Supporting Information

\title{
Cellulose-Based Superhydrophobic Surface Decorated with Functional Groups Showing Distinct Wetting Abilities to Manipulate Water Harvesting
}

\begin{abstract}
Wei Huang, ${ }^{\dagger}$ Xiangyu Tang, ${ }^{\dagger}$ Zhe Qiu, ${ }^{\dagger}$ Wenxin Zhu, ${ }^{\dagger}$ Yonggui Wang, ${ }^{*}, \dagger$ You-Liang Zhu, ${ }^{*}$ Zefang Xiao, ${ }^{\dagger}$ Haigang Wang, ${ }^{\dagger}$ Daxin Liang, ${ }^{\dagger}$ Jian Li, ${ }^{\dagger}$ Yanjun Xie $*, \dagger, \S$
\end{abstract}

${ }^{\dagger}$ Key Laboratory of Bio-based Material Science and Technology (Ministry of Education), College of Material Science and Engineering, Northeast Forestry University, Hexing Road 26, Harbin 150040, P. R. China

$\$$ State Key Laboratory of Polymer Physics and Chemistry, Changchun Institute of Applied Chemistry, Chinese Academy of Sciences, Changchun 130022, P. R. China

$\S$ Engineering Research Center of Advanced Wooden Materials (Ministry of Education), College of Materials Science and Engineering, Northeast Forestry University, Hexing Road 26, Harbin 150040, P. R. China

\section{Corresponding Author}

*Email: wangyg@nefu.edu.cn (Y. Wang);

*Email: yxie@nefu.edu.cn (Y.Xie). 
Movie S1. Optical microscopy showing water harvesting performance on SU-CNPs.

Movie S2. Optical microscopy showing water harvesting performance on S-ODT.

Movie S3. Optical microscopy showing water harvesting performance on S-PFDT.

Movie S4. Optical microscopy showing water harvesting performance on S-MH.

Movie S5. Optical microscopy showing water harvesting performance on S-MPA.

Movie S6. Optical microscopy showing water harvesting performance on S-CA.

Movie S7. MD Simulation for water harvesting on H-SPhi.

Movie S8. MD Simulation for water harvesting on SH-Pho.

Movie S9. MD Simulation for water harvesting on SH-MPhi.

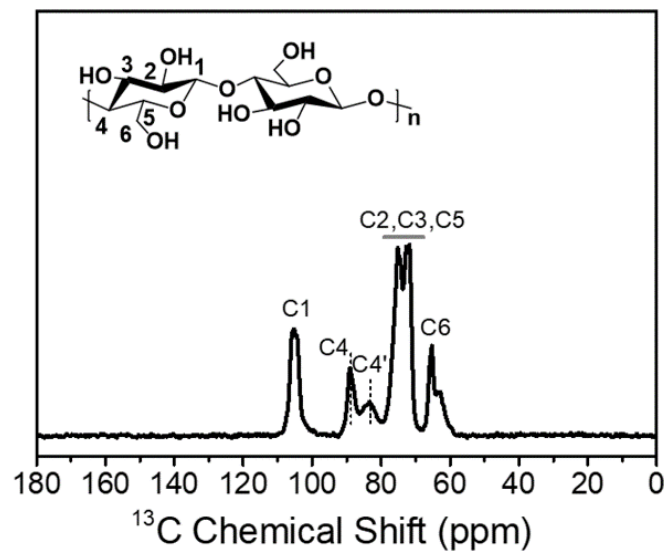

Figure S1. Solid-state ${ }^{13} \mathrm{C}$ NMR spectra of MCC. 

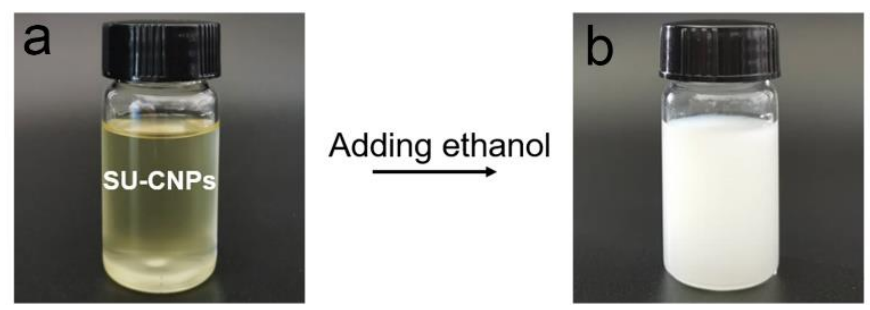

Figure S2. (a) THF suspension of SU-CNPs before adding ethanol. (b) Suspension of SU-CNPs after adding ethanol (THF/ethanol volume ratio: 1/5).

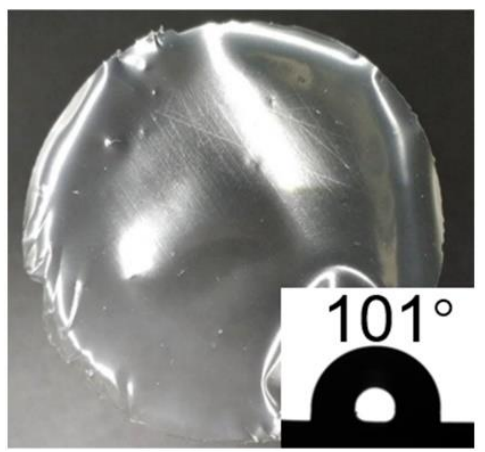

Figure S3. SU-CNPs film dried from its THF suspension.
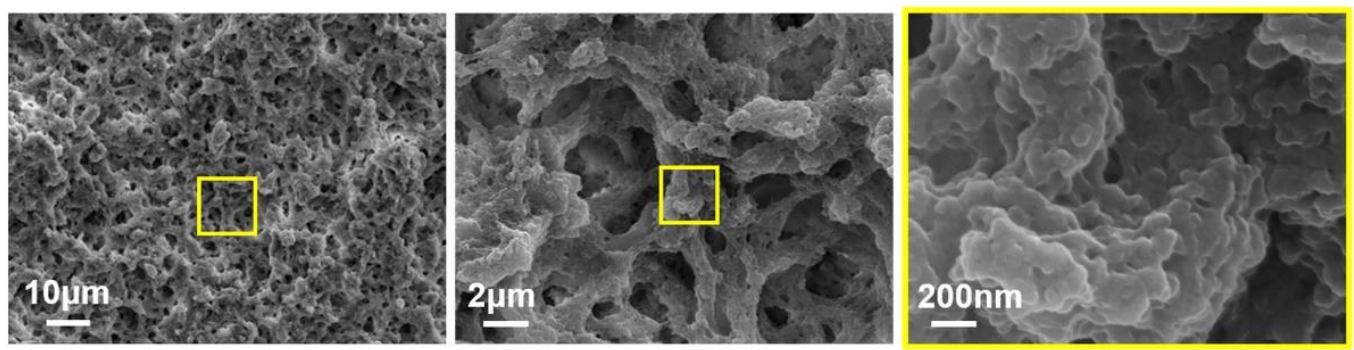

Figure S4. SEM images of the superhydrophobic surface via spraying SU-CNPs suspension in the mixture of THF/ethanol. 


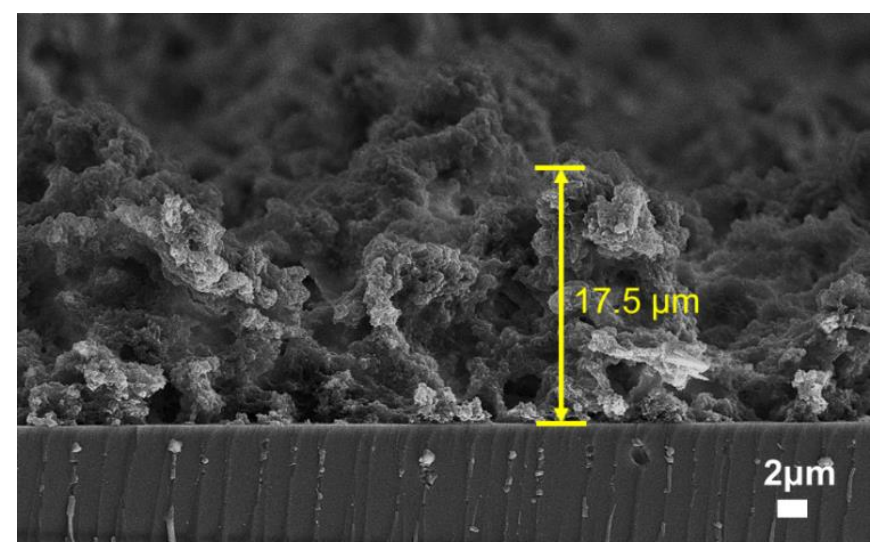

Figure S5. The SEM image of the cross section of the superhydrophobic surface via spraying SUCNPs suspension on glass slide. 


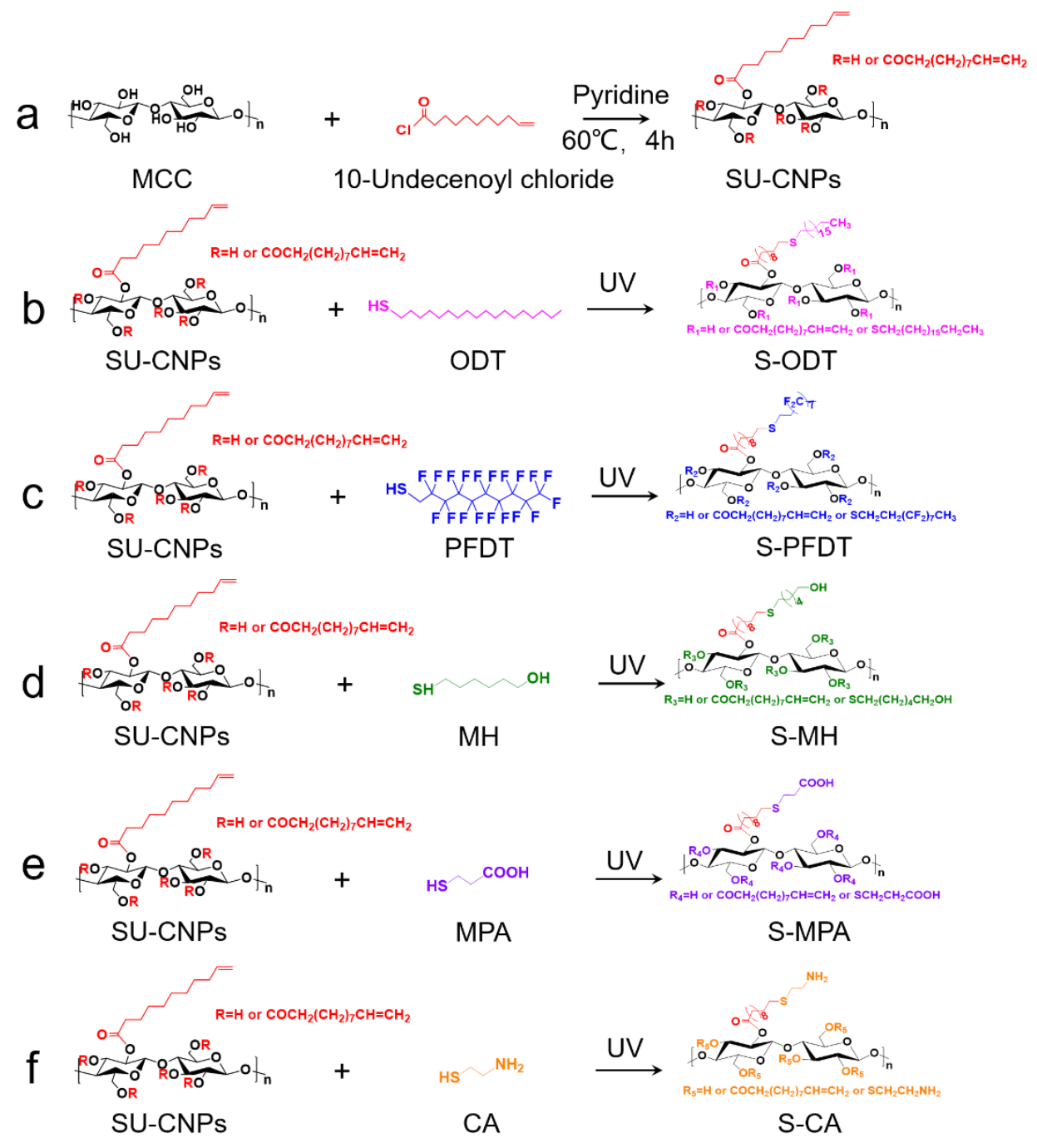

Figure S6. (a) Chemical reaction for the synthesis of SU-CNPs. (b-f) Chemical reactions for surface modification to form (b) S-ODT, (c) S-PFDT, (d) S-MH, (e) S-MPA and (f) S-CA. 
Table S1. Elemental contents of N, F and S determined by EDS

\begin{tabular}{cccc}
\hline Surfaces & N/At $\%$ & F/At\% & S/At\% \\
\hline SU-CNPs & 0 & 0 & 0 \\
S-ODT & 0 & 0 & 1.33 \\
S-PFDT & 0 & 3.3 & 0.18 \\
S-MH & 0 & 0 & 0.96 \\
S-MPA & 0 & 0 & 2.11 \\
S-CA & 5.12 & 0 & 0.91 \\
\hline
\end{tabular}
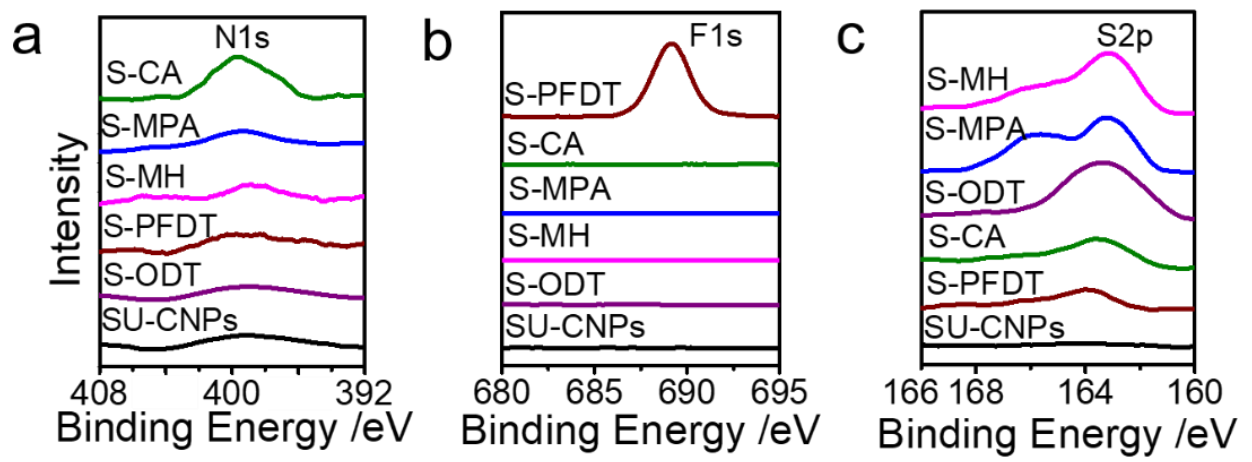

Figure S7. XPS spectra of modified surfaces. (a) N 1s spectrum, (b) F 1s spectrum, (c) S 2p spectrum.

Table S2. Elemental contents of N, F and S determined by XPS

\begin{tabular}{cccc}
\hline Surfaces & N/At $\%$ & F/ At $\%$ & S/ At \% \\
\hline SU-CNPs & 0 & 0 & 0 \\
S-ODT & 0 & 0 & 2.12 \\
S-PFDT & 0 & 13.42 & 0.64 \\
S-MH & 0 & 0 & 2.71 \\
S-MPA & 0 & 0 & 4.01 \\
S-CA & 2.81 & 0 & 1.30 \\
\hline
\end{tabular}




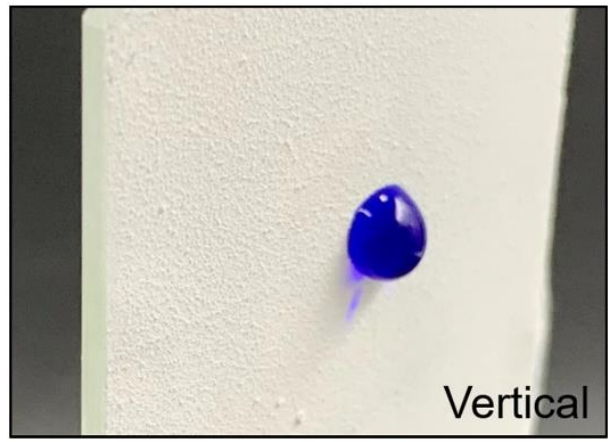

Figure S8. Photo image of a dyed water droplet on S-MPA surface vertically.
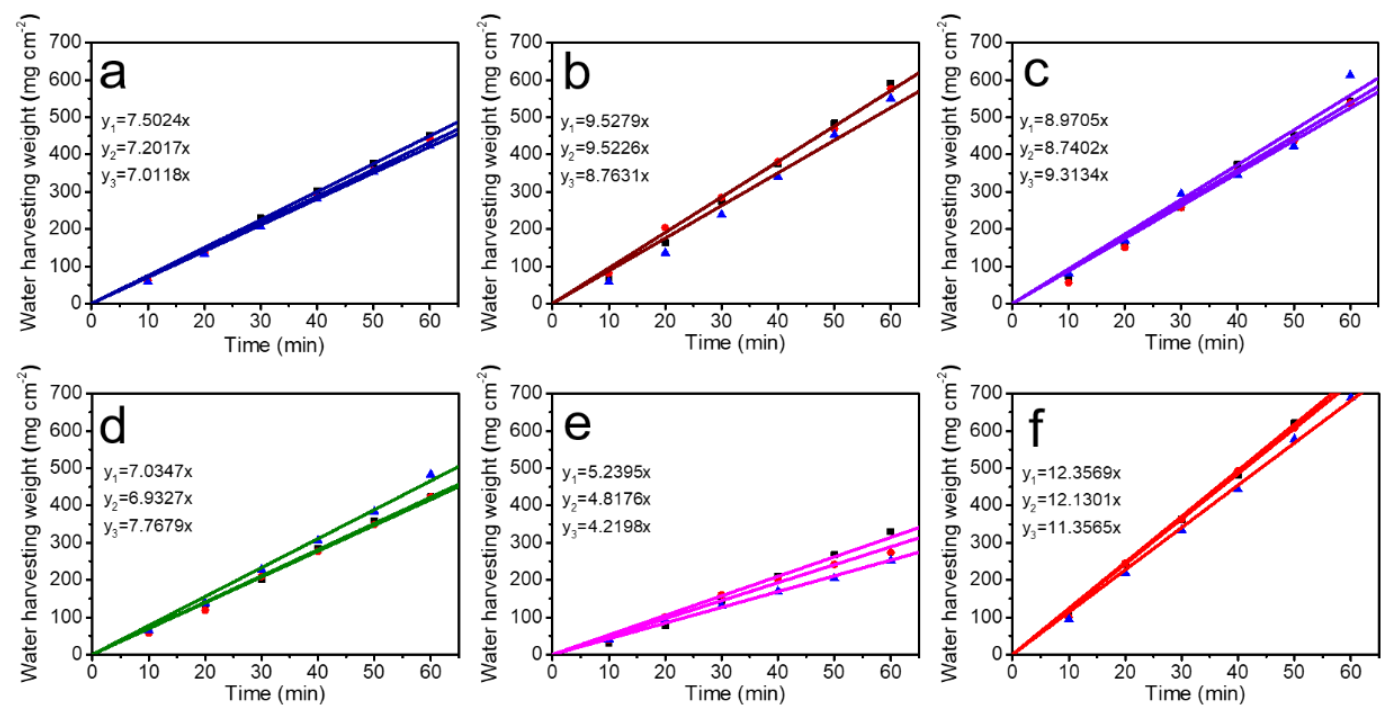

Figure S9. Water harvesting weight for each sample along with the harvesting time. (a) SU-CNPs, (b) S-ODT, (c) S-PFDT, (d) S-MH, (e) S-MPA, (f) S-CA. The coefficients of determination $\left(\mathrm{R}^{2}\right)$ for all linear fits are higher than 0.99 . 


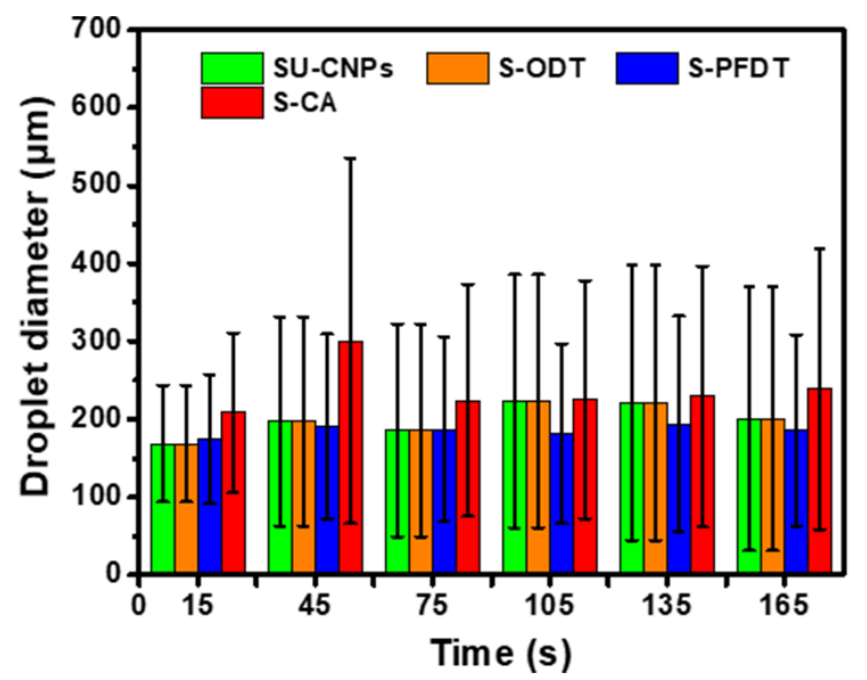

Figure S10. The variation of the average droplet diameter for different surfaces within 165s (no data for S-MH and S-MPA due to filmwise collection).

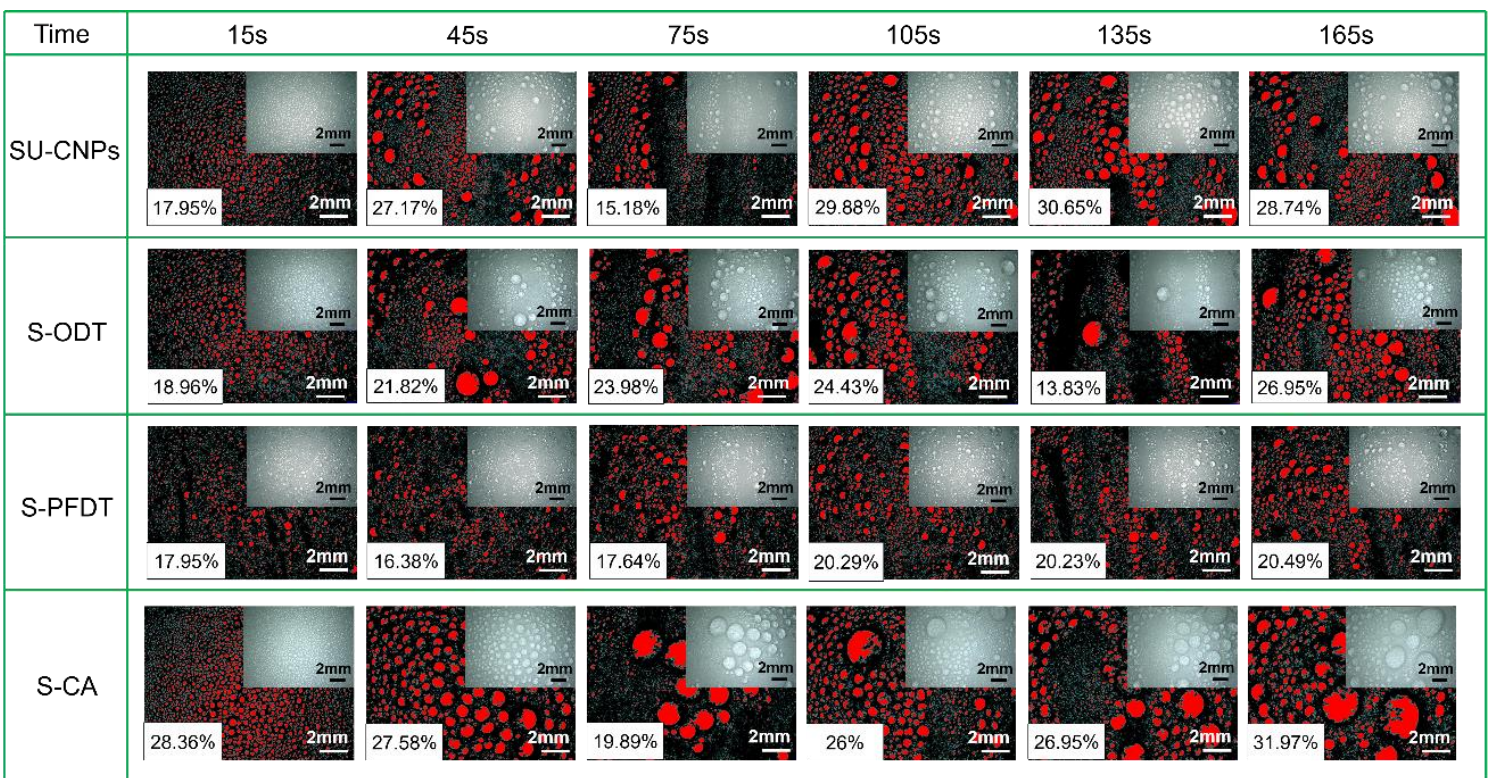

Figure S11. The surface coverage of different surfaces within 165s (no data for S-MH and S-MPA due to filmwise collection). 

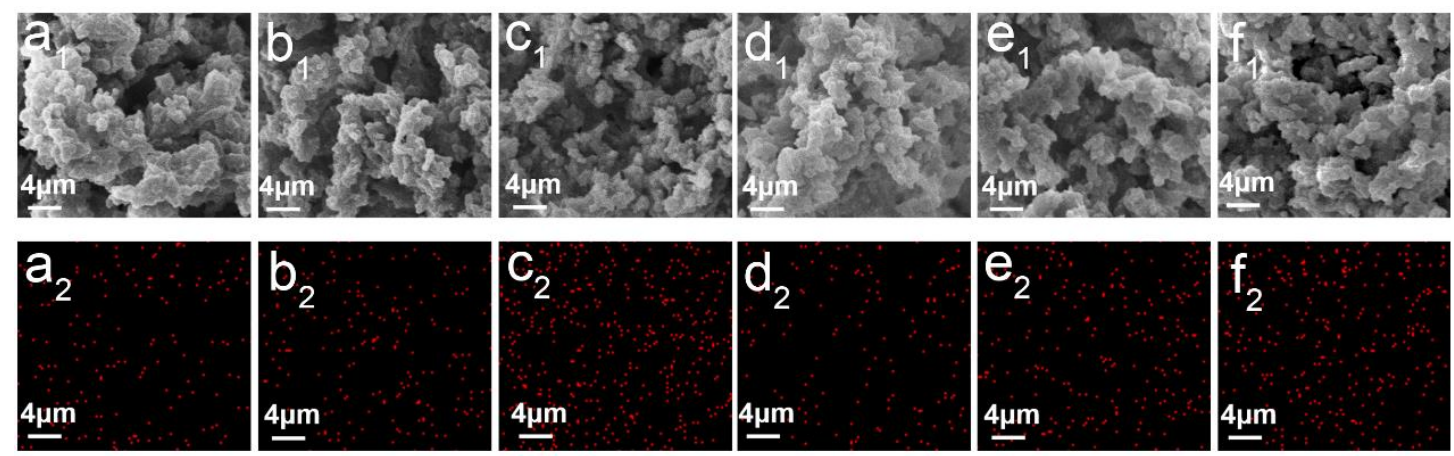

Figure S12. SEM images of ( $\left.a_{1}\right)$ S-MPA-0.05, $\left(b_{1}\right)$ S-MPA-0.15, $\left(c_{1}\right)$ S-MPA-0.3, $\left(d_{1}\right)$ S-CA-0.05, $\left(\mathrm{e}_{1}\right)$ S-CA-0.15 and $\left(\mathrm{f}_{1}\right)$ S-CA-0.3, and corresponding EDS element maps of S for $\left(\mathrm{a}_{2}\right) \mathrm{S}-\mathrm{MPA}-$ 0.05, $\left(\mathrm{b}_{2}\right)$ S-MPA-0.15, $\left(\mathrm{c}_{2}\right)$ S-MPA-0.3, $\left(\mathrm{d}_{2}\right)$ S-CA-0.05, $\left(\mathrm{e}_{2}\right)$ S-CA-0.15 and $\left(\mathrm{f}_{2}\right)$ S-CA-0.3, respectively.
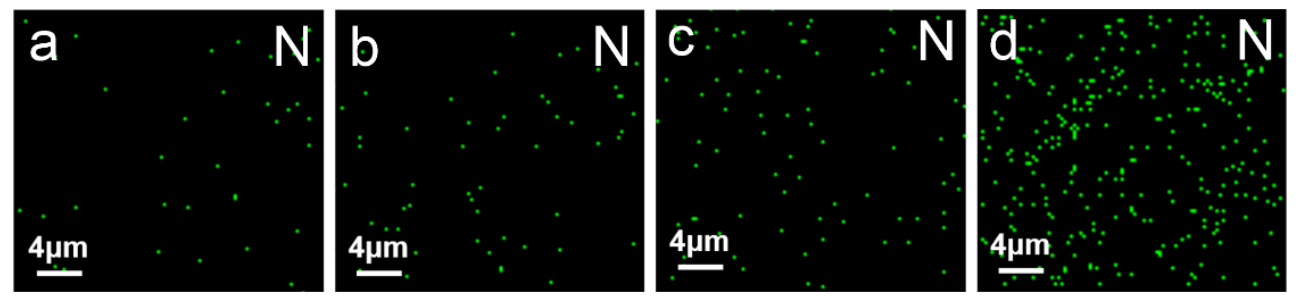

Figure S13. EDS element maps of $\mathrm{N}$ for (a) S-CA-0.05, (b) S-CA-0.15, (c) S-CA-0.3 and (d) SCA-0.5.

Table S3. Elemental contents of $\mathrm{N}$ and $\mathrm{S}$ determined by XPS

\begin{tabular}{|c|c|c|}
\hline Surfaces & $\mathrm{S} \%$ & $\mathrm{~N} \%$ \\
\hline S-MPA-0.05 & 0.88 & 0 \\
\hline S-MPA-0.15 & 1.39 & 0 \\
\hline S-MPA-0.3 & 3.64 & 0 \\
\hline S-MPA-0.5 & 4.01 & 0 \\
\hline S-CA-0.05 & 0.24 & 1.33 \\
\hline S-CA-0.15 & 0.25 & 1.46 \\
\hline S-CA-0.3 & 0.48 & 1.94 \\
\hline S-CA-0.5 & 1.30 & 2.81 \\
\hline
\end{tabular}




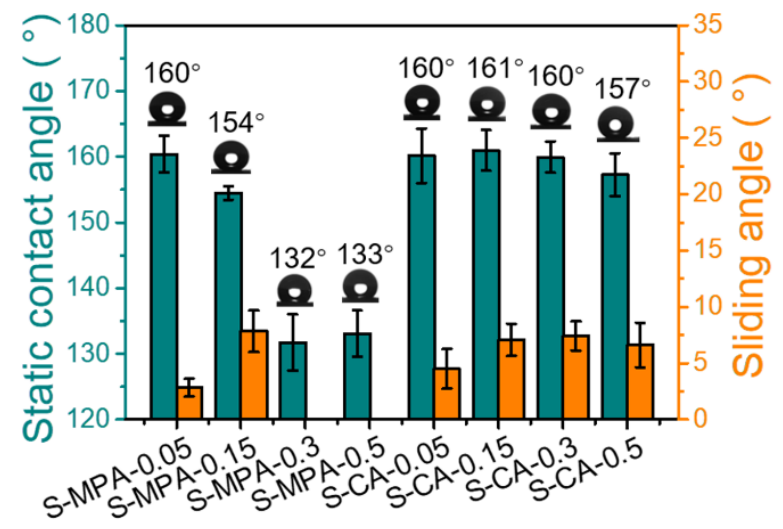

Figure S14. Static contact angles and sliding angles of S-MPA-0.05, S-MPA-0.15, S-MPA-0.3, S-CA-0.05, S-CA-0.15 and S-CA-0.3. (The water droplets can keep satble vertically on S-MPA0.3 and S-MPA-0.5)

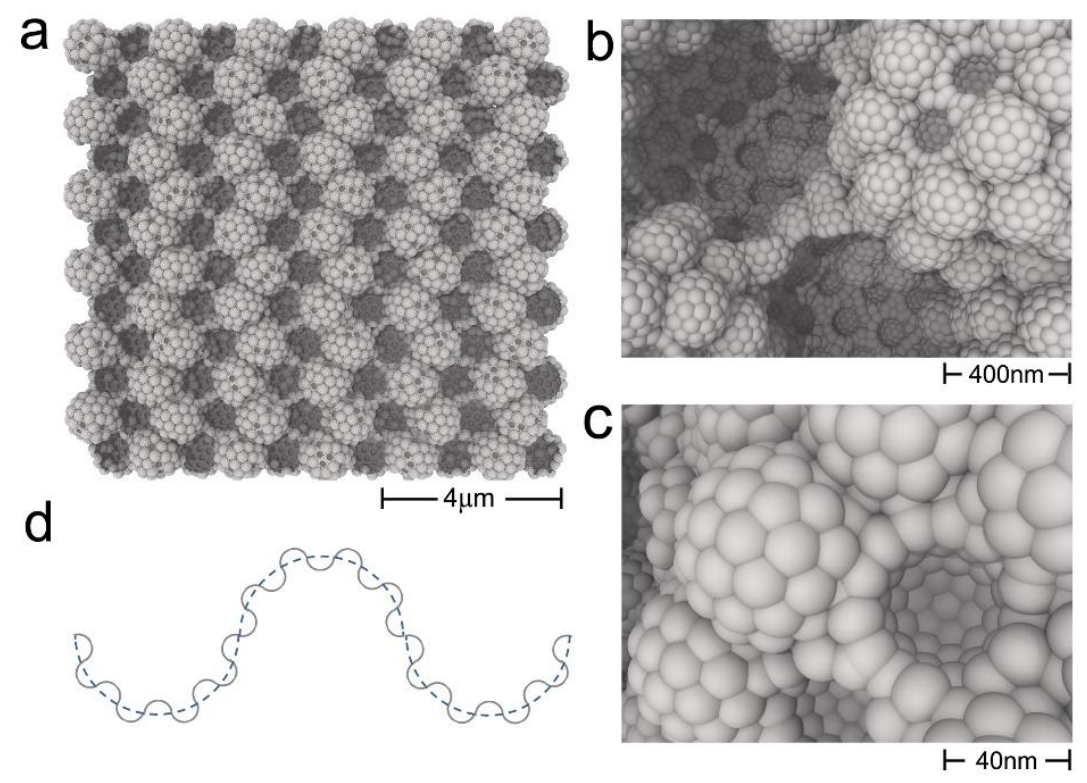

Figure S15. Simulation model of SU-CNPs superhydrophobic surface. (a) Surface with a fractal structure of hemisphere. (b) and (c) Local structure that is zoomed in. (d) Illustration of fractal structure. 